\title{
Robust-control using model-error-feedback CDOB and DOB under variable-time-delay
}

\author{
Masato Koyama \\ Dept. of Electrical and Electronic Engineering \\ Mie University \\ Mie, Japan \\ koyama@elec.mie-u.ac.jp
}

\author{
Yosuke Asano \\ Dept. of Electrical and Electronic Engineering \\ National Institute of Technology, Kisarazu College \\ Chiba, Japan \\ asano@e.kisarazu.ac.jp
}

\author{
Tomoyuki Shimono \\ Dept. of Electrical and Computer Engineering \\ Yokohama National University \\ Kanagawa, Japan \\ shimono@ynu.ac.jp
}

\begin{abstract}
This paper presents the robustness improvement of the Communication-Disturbance-OBserver (CDOB) based on Lyapunov-Krasovskii functional(LKF), and proposes the Network-based-Control-System (NCS) using the LKF based CDOB and a local-side DOB. The CDOB is a compensation method for a output signal includes a communication delay. By using LKF, a steady-state output-estimation-error of the CDOB under variable time-delay and parameter-perturbations is suppressed. However, the latest studies have not shown the robustness to the disturbance-input. In this paper, the frequencyweight of CDOB to the disturbance is introduced to the design of the LKF based CDOB, to suppress the error. From a calculated error-characteristic and a numerical simulation, the account of the weight is validated. Additionally, implementing a disturbance observer in the local (controlled object) side, the robustness of the NCS is improved much in the numerical simulation.

Index Terms-Communication disturbance observer (CDOB), Lyapunov-Krasovskii functional (LKF), network based control system
\end{abstract}

\section{INTRODUCTION}

Nowadays, in industrial fields, the information technology via Ethernet networks has been introduced to factoryautomations, and gathered attentions. Big data from the any sensors are utilized to analyze or manage systems. Some countries have proposed the factory-standards based on "Information and Communication Technology (ICT)" and "Internet of Things (IoT)" such as Industrie 4.0 (Germany) [1].

To achieve a control system that is applicable for IoT, various control methods of Networked-Control-Systems (NCSs) have been proposed. In the NCS, communication between a controller and a target object is via the networks (LAN cable, WiFi and so on). Then, it is possible to set the controller far from the controlled object. Applications of this system are distributed power-grid control, remote-car-driving, remote bilateral-control [2] [3], wireless motion-control of actuators [4] [5], and so on [6]. These applications require a feedback controller via networks. On the other hand, a conventional remote-control-system generally consists of open- loop/feedforward control. A distributed operator sends a reference signal to a controller installed at a target side. Using the reference, a local feedback-loop is implemented. Therefore, it is impossible to realize a system such as the bilateral controls, because it needs feedback from both of a master and slave. In other words, for a system need a synchronized control, the conventional system is not applicable. Contrary to this, NCS deals a system has a communication delay inserted to a control input and feedback signal. However, the delay affects the characteristic equation, then there is possibility that the system is destabilized.

To solve the destabilization caused by time-delay, some compensation methods have been proposed [8]- [14]. The first method is "smith-method", this uses a time-delay model for feedforward controller. Although the precise delay-model is required, the delay-term in the characteristic equation is completely suppressed. Recently, some modified smith-methods are presented, that is the adaptive or identification methods are applied to the delay-model calculation [7]. The second method is "communication disturbance observer", that estimates a disturbance caused by the delay [8]. Using the disturbance observer, it is possible to compensate the effect of constant delay as like the smith-method, without any delay-models. However, when variable delay is inserted, there is a problem about steady-state estimation-error of CDOB. The third method is $\mathrm{H} \infty$ control based on Lyapunov-Krasovskii Functional (LKF), that guarantee the stability consider the variable delay [9][10]. Lyapunov-Krasovskii functional is an extended Lyapunov stability criterion considering time-delay. By assuming the upper bound of the delay, the LKF can guaranties the stability that is regardless of the delay variation. Therefore, introducing LKF to Linear Matrix Inequality (LMI), it is possible to design $\mathrm{H} \infty$ controller satisfies the LKF [11]- [12].

The account of LKF is that it is possible to design a stable NCS, if the expected upper bound of the delay is given. Whereas, it is difficult to decrease the conservative of the 


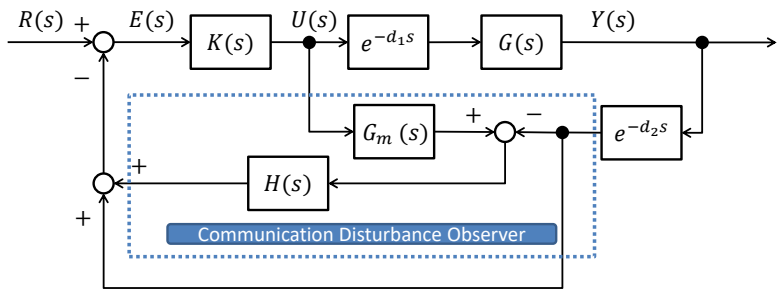

Fig. 1: The NCS using the CDOB.

$\mathrm{H} \infty$ controller. As one of solutions, it has been proposed that CDOB modified by using the LKF based design [13] [14]. The papers presents a design method of CDOB considering LKF, it consists of $\mathrm{H} \infty$ state-observer based on the LMI. Then, from the LKF based CDOB and plant model, the mixed $\mathrm{H} 2 / \mathrm{H} \infty$ controller for NCS is designed to guarantee the stable. By using the mixed $\mathrm{H} 2 / \mathrm{H} \infty$ controller, the conservative of feedback controller is decreased. Modifying CDOB based on LKF, this method has achieved to suppress the steady-state error of CDOB under variable delay. Additionally, in the paper, the robustness to parameter-perturbations has been confirmed from the experiment. Meanwhile, to disturbance-inputs, these papers have not clarified the robustness.

Hence, this paper presents the robustness of the LKF based CDOB to disturbance inputs, and improve the robustness by introducing an controlled-object side disturbance observer. In this paper, the mathematical frequency-analysis of the robustness is derived and computed. Then, a frequency-weight of the LKF based CDOB is discussed. Additionally, the controlledobject side disturbance-observer is introduced to improve the robustness. Finally, the numerical simulations of NCS using the LKF based CDOB are shown to validate the account of the proposed NCS.

\section{LKF BASED CDOB}

\section{A. Communication Disturbance OBserver (CDOB)}

$\mathrm{CDOB}$ has been proposed to compensate the destabilization caused by time-delay without time-delay model. In CDOB, the effect of the time-delay is dealt as a disturbance input. Due to this, the fundamental structure is similar to Disturbance OBservers (DOB). The block-diagram of the CDOB is shown in Fig. 1.

Here, as shown in (1) and (2), we define the system that delay inserted.

$$
\begin{aligned}
\dot{x}(t) & =\mathbf{A} x(t)+\mathbf{B} u\left(t-d_{1}\right) \\
y(t) & =\mathbf{C} x\left(t-d_{2}\right)
\end{aligned}
$$

The transfer function of the output in the plant-side is shown as (3).

$$
Y(s)=G(s) U(s) e^{-d_{1} s}
$$

where, $G(s)$ is a plant model $\left(C(s I-A)^{-1} B\right)$.
From (3), the feedback system of (3) has the characteristic shown as (4).

$$
\frac{Y(s)}{R(s)}=\frac{K(s) G(s) e^{-d_{1} s}}{1+K(s) G(s) e^{-d s}}
$$

where, $R(s)$ is a reference, $K(s)$ is a controller, and $d$ is $d_{1}+d_{2}$.

Due to that the characteristic equation $1+K(s) G(s) e^{-d s}$ contains the delay, the system is easy to be unstable. To compensate the time-delay effect, it is necessary to remove the delay term from the equation. Equivalently, if the delay in the feedback is removed, the compensation is achieved. For the delay rejection, CDOB estimates the disturbance input caused by the delay as shown in (5).

$$
\hat{D}_{\text {com }}(s)=H(s)\left(U(s)-G_{m}^{-1}(s) Y(s)\right)
$$

where, $\hat{D}_{\text {com }}$ is an estimated communication-disturbance, $H(s)$ is a filter of CDOB, and $G_{m}(s)$ is a nominal plant model, respectively.

From (5), if the parameter error is nothing, the $\hat{D}_{\text {com }}$ is given as (6).

$$
\begin{aligned}
\hat{D}_{c o m}(s) & =H(s)\left(U(s)-G_{m}^{-1}(s) G(s) U(s) e^{-d s}\right) \\
& =H(s) U(s)\left(1-e^{-d s}\right)
\end{aligned}
$$

Then, by using the $\hat{D}_{\text {com }}$, it is possible to compensate the feedback error caused by the delay.

$$
G_{m}(s) \hat{D}_{c o m}(s)=H(s) G_{m}(s) U(s)\left(1-e^{-d s}\right)
$$

Let us assume the cutoff frequency of $H(s)$ is enough high, the delay term is completely removed from the output as shown in (8).

$$
\begin{aligned}
Y_{c} & =Y(s)+G_{m}(s) \hat{D}_{c o m}(s) \\
& =G(s) U(s) e^{-l s}+H(s) G_{m}(s) U(s)\left(1-e^{-d s}\right) \\
& \simeq G(s) U(s)\left(e^{-l s}+1-e^{-l s}\right)=G(s) U(s)
\end{aligned}
$$

As a result, in the specified condition that the delay is constant and parameter error is nothing, $\mathrm{CDOB}$ can guarantee the stability of the system. However, if variable delay is inserted or parameter error exist, the recent papers report the CDOB has steady-state estimation error of the $G_{m}(s) U(s)\left(1-e^{-d s}\right)$. This error is equivalent with the reference variation, owing to this, the controller can not suppress it. To solve this problem, the $\mathrm{H} \infty$ state-observer of CDOB based on LKF has been proposed.

\section{B. LKF based $C D O B$}

Lyapunov-Krasovskii Functional (LKF) is one of Lyapunov functional extended to consider time-delay. The stability is guaranteed by Lyapunov stability criterion. By using Linear Matrix Inequality (LMI) expression of the LKF, it is possible 
to design the $\mathrm{H} \infty$ control that is stable under variable delay. A LKF candidate is shown as (9).

$$
\begin{aligned}
V(t)= & x_{m}^{T}(t) Z_{m} x_{m}(t)+\int_{t-d(t)}^{t} x_{m}^{T}(\tau) R_{m} x_{m}(\tau) d \tau \\
& +\int_{-\bar{d}}^{0} \int_{t+\tau}^{t} \dot{x}_{m}^{T}(\alpha) Q_{m} \dot{x}_{m}(\alpha) d \alpha d \tau
\end{aligned}
$$

where, $Z_{m}, R_{m}$, and $Q_{m} \in \mathbb{R}^{n \times n}$ are symmetric-positive matrices, and $d(t)$ is variable time delay assumed as a follow; $\bar{d}$ is upper bound of the delay, and $\delta$ is a variation rate of the delay.

$$
0 \leq d(t) \leq \bar{d} \leq \infty, \dot{d}(t) \leq \delta \leq \infty
$$

The first term in the light-hand side of the (9) is often used as general Lyapunov-function, the second and third term can consider an integral of the state variation caused by the time delay. Therefore, to guarantee the stability under the variable delay, the terms have to satisfy the Lyapunov stability criterion.

In many papers, the $\mathrm{H} \infty$ controllers based on the LKF have been proposed. According to the simulations and experiments, the LKF is validated enough. Although the controller achieves the strong stability, the design process is complicated. Due to this, it is not easy to decrease the conservative of the controllers. On the other hand, the papers [13], [14] have proposed the $\mathrm{CDOB}$ consists of $\mathrm{H} \infty$ state observer based on LKF. Additionally, the controller considers the CDOB is also proposed. By this method, it is possible to independent design the state-observer and controller guarantee the stability under the variable delay.

Here, let us present the CDOB under the variable delay as (11). The block-diagram of the NCS using this CDOB is shown as Fig. 2.

$$
\begin{aligned}
\dot{x}_{m}(t)= & A_{m} x_{m}(t)+B_{m} u(t) \\
& +K_{o}\left(-C_{m} x_{m}(t-d(t))+y(t)\right) \\
z(t)= & -C_{m} x_{m}(t-d(t))+y(t)
\end{aligned}
$$

where, $x_{m} \in \mathbb{R}^{n \times 1}$ is state-variable of the observer, $A_{m} \in$ $\mathbb{R}^{n \times n}, B_{m} \in \mathbb{R}^{n \times q}$ and $C_{m} \in \mathbb{R}^{p \times n}$ are the system, input and output matrices, $K_{o} \in \mathbb{R}^{n \times p}$ is the observer gain, $y \in \mathbb{R}^{p \times 1}$ is the plant output, and $z \in \mathbb{R}^{p \times 1}$ is the estimation error. In the design process of the $K_{o}$, the input $u(t)$ is neglected. The structure of frequency-weight $G_{f w}$ shown in Fig. 2 is LPF in [13], [14], and discussed in III-A.

If the $\mathrm{H} \infty$ norm of the gain from $y$ to $z$ is less than a desired value $\gamma$, it can design the CDOB that is stable and the steady-state error is suppressed. According to the paper, from

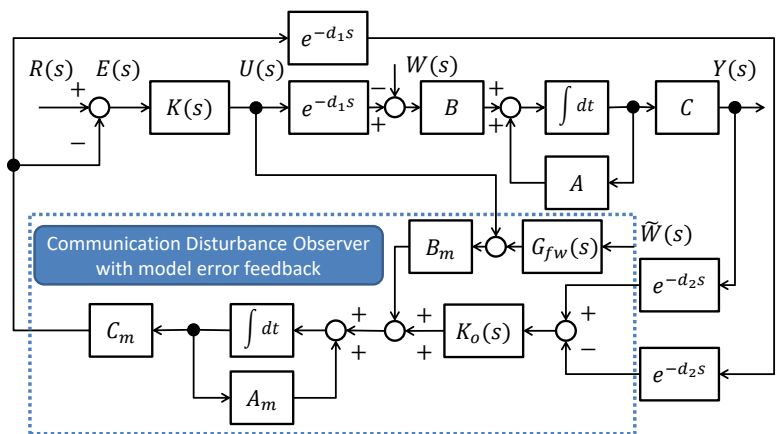

Fig. 2: The NCS using the model-error feedback CDOB [13].

the LMI as (12), it is possible to conduct the CDOB.

$\left[\begin{array}{cccccc}\Omega_{m 11} & \Omega_{m 12} & -P_{m 1} & M & \Omega_{m 15} & 0 \\ \Omega_{m 12}^{T} & \Omega_{m 22} & -P_{m 2} & 0 & \Omega_{m 25} & C_{m}^{T} \\ -P_{m 1}^{T} & -P_{m 2}^{T} & -\bar{d}^{-1} Q_{m} & 0 & 0 & 0 \\ M^{T} & 0 & 0 & -\gamma^{2} I_{1} & \Omega_{m 45} & I_{2} \\ \Omega_{m 15}^{T} & \Omega_{m 25}^{T} & 0 & \Omega_{m 45}^{T} & \Omega_{m 55} & 0 \\ 0 & C_{m} & 0 & I_{2} & 0 & -I_{3}\end{array}\right]<0$

$M=Z_{m} K_{o}, \Omega_{m 11}=A_{m}^{T} Z_{m}+Z_{m}^{T} A_{m}+P_{m 1}+P_{m 1}^{T}+R_{m}$ $\Omega_{m 12}=-M C_{m}-P_{m 1}+P_{m 2}^{T}, \Omega_{m 15}=\bar{d} A_{m}^{T} Z_{m}$

$\Omega_{m 22}=-(1-\delta) R_{m}-P_{m 2}-P_{m 2}, \Omega_{m 25}=\bar{d}\left(M C_{m}\right)^{T}$

$\Omega_{m 45}=\bar{d} M^{T}, \Omega_{m 55}=\bar{d}^{-1} \Omega_{m}-2 Z_{m}$

where, $P_{m 1}$ and $P_{m 2}$ are square positive matrices $\in \mathbb{R}^{n \times n}$, and $I_{1}, I_{2}$ and $I_{3}$ are unit matrices $\in \mathbb{R}^{p \times p}$, respectively.

In this paper, to solve the LMI, the solver "SeDuMi" is used. Then, the CDOB based on LKF is given by (11) and the LMI. Additionally, by introducing a frequency-weight as other $\mathrm{H} \infty$ controls, it is possible to tune the robustness and the estimation performance. However, according to the paper [13], the design of weight is not discussed obvious. Due to this, this paper presents a proper frequency-weight and improve the robustness of the NCS using the LKF based CDOB.

\section{PRoposed NCS: LKF BASED CDOB WiTH LOCAL-SIDE DOB}

In this section, for the LKF based CDOB, a target-system and a frequency-weight of a disturbance-input are discussed firstly. From the bode-plots of the $\mathrm{CDOB}$, the robustness of the estimation is discussed. Secondary, by introducing the Disturbance Observer in the plant-side, this paper targets the improving the robustness of the NCS.

In this paper, the plant-system is defined as a simplified linear-motor model. The model contains only the motor dy- 
TABLE I: A comparison condition of the frequency-weight.

\begin{tabular}{|c|c|c|c|c|}
\hline & case 1 & case 2 & case 3 [13], [14] & case 4 \\
\hline \multirow{2}{*}{ weight } & 0 & 1 & $\frac{200 \pi \times 10^{15}}{s+200 \pi}$ & $\frac{200 \pi \times 10^{15}}{s(s+200 \pi)}$ \\
\hline
\end{tabular}

namics as shown in (13).

$$
\begin{aligned}
\dot{x} & =A x+B u \\
y & =C x \\
x & =\left[\begin{array}{ll}
l & i
\end{array}\right]^{T}, u=\left[\begin{array}{ll}
f_{i} & f_{w}
\end{array}\right]^{T}, A=\left[\begin{array}{cc}
0 & 1 \\
0 & 0
\end{array}\right] \\
B & =\left[\begin{array}{ll}
B_{i} & B_{w}
\end{array}\right], B_{i}=\left[\begin{array}{c}
0 \\
\frac{1}{M}
\end{array}\right], B_{w}=\left[\begin{array}{c}
0 \\
\frac{-1}{M}
\end{array}\right], C=\left[\begin{array}{ll}
1 & 0
\end{array}\right]
\end{aligned}
$$

where, $l$ and $l$ are the mover position and velocity, $M$ is a mass of the mover and equals to $5 \mathrm{~kg}$ in this paper, and $f_{i}$ and $f_{w}$ are the input and disturbance force, respectively.

\section{A. frequency-weight of the $C D O B$}

In this subsection, the robustness to the disturbance-input of the CDOB is discussed from the bode-plot. For improvement of the robustness, it is necessary to introduce a frequencyweight of the disturbance to the LMI. Due to this, the plantsystem is modified to the extended system as (15).

$$
\begin{aligned}
& \dot{x}_{e x}(t)=A_{e x} x_{e x}(t)+B_{e x} u(t) \\
& x_{e x}=\left[\begin{array}{lll}
l & i & x_{f w}
\end{array}\right]^{T}, u=\left[\begin{array}{ll}
f_{i} & f_{w}
\end{array}\right]^{T} \\
& A_{e x}=\left[\begin{array}{cc}
A & B_{w} C_{f w} \\
0 & A_{f w}
\end{array}\right], B_{e x}=\left[\begin{array}{cc}
B_{i} & 0 \\
0 & B_{f w}
\end{array}\right]
\end{aligned}
$$

where, $x_{f w}$ is an internal-state of the frequency-weight for the disturbance, and $A_{f w}, B_{f w}$ and $C_{f w}$ are a system, input, and output matrices of the frequency-weight, respectively.

Introducing the frequency-weight, in the LMI, it is possible to consider the $\mathrm{H} \infty$ norm for the estimation-error given by the disturbance. In this subsection, four cases are shown to validate the estimation-error variation given by the weight. The each frequency-weight of the cases is shown in Table I. The papers [13], [14] used a LPF such as case 3, for the weight. Then, a robustness to parameter-perturbation was improved. However, depending on the suppression performance, some steady-state error would be remained. Therefore, this paper proposes introducing integral factor such as case 4 , to much decrease the sensitivity in low-frequency region. As a result, the bode-plots are given as shown in Fig. 3 .

From the Fig. 3, the peak gain of the estimation-error given by the output and the disturbance is shown in Table II. From the Table II, although these characteristics does not consider the relationship between the delays and the estimation-error, it is possible to confirm that the case 1,2 and 3 have higher gain than case 4 . Especially, focusing on Fig. $3 \mathrm{~b}$, the three cases amplify the error given by the disturbance. On the other hand, at the peak, the case 4 can suppress three times more estimation-errors given by the disturbance than case 3 .

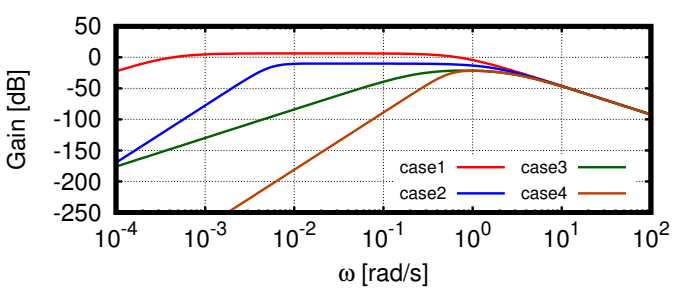

(a) output to estimation-error (per $1 \mathrm{~m}$ )

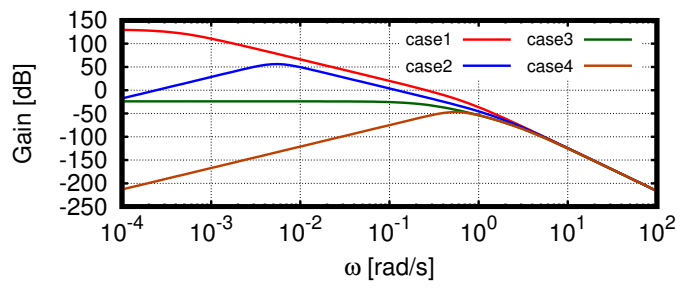

(b) disturbance-input to estimation-error (per 1N)

Fig. 3: Estimation-error characteristics of the LKF based CDOB; the error system is given by the (11) that the delays are assumed as $(d(t)=0)$.

TABLE II: The peak gains of the estimation-error shown in Fig. 3

\begin{tabular}{|c||c|c|c|c|}
\hline $\begin{array}{c}\text { Peak gain[dB] } \\
\text { (output) }\end{array}$ & 2.75 & -4.73 & -9.22 & -9.41 \\
\hline $\begin{array}{c}\text { Peak gain[dB] } \\
\text { (disturbance) }\end{array}$ & 56.5 & 24.4 & -10.4 & -20.3 \\
\hline
\end{tabular}

It means that, even if there is disturbance-inputs, the stability (convergence) holds.

Depending on the amplitude of disturbance, it is difficult to neglect the estimation-error. However, there is a limit of the robustness improvement by the frequency-weight, because the compensation performance is finally designed by the LMI considers the stability under delays. Therefore, in this paper, a disturbance-observer (DOB) is implemented in the plant side.

\section{B. combination with local- $D O B$}

Introducing a local DOB, the disturbance response in the output is suppressed. Then, the amplitude of the estimation error given by the disturbance is also suppressed. The DOB is constructed as shown in Fig. 17.

$$
\hat{W}(s)=\frac{g}{s+g}\left(V(s)-G^{-1}(s) Y(s)\right)
$$

Then, the proposed NCS is constructed as shown in Fig. 4.

This paper proposes the NCS shown in Fig. 17. In [13], [14], the combination of the LKF-based CDOB and local DOB has not been discussed. To validate the account the NCS of the authors proposal, next section discusses a numerical simulation of the NCS.

\section{NUMERICAL SIMULATION}

This section presents a numerical simulation of the NCS using the linear-motor model. Network models for I/O are 


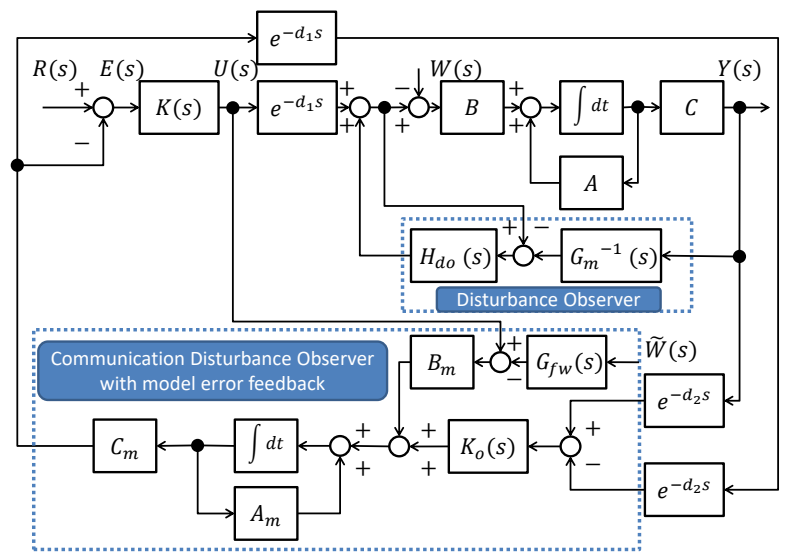

Fig. 4: The structure of the proposed NCS.

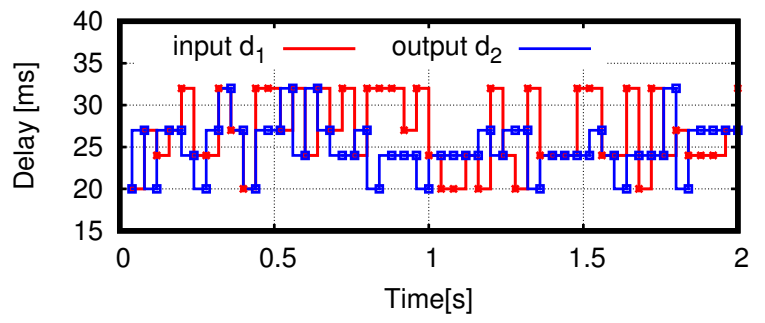

Fig. 5: The input and output time-delay between $0 \mathrm{~s}-2 \mathrm{~s}$; the duration of points means the communication-period.

generated by referring to the measurement of Bluetooth v2.0 [15]. The example of the delay is shown in Fig. 5.

In this simulation, the LKF based CDOB using the case 3 and case 4 of the frequency-weight is compared by the tracking and disturbance response. Additionally, to validate the account of the proposed NCS, the LKF based CDOB under implementing local-DOB implementing is compared with them. Figure $6-9$ show the position responses of the plant (linear-motor) and the estimated by CDOB. Since 0 s, the position reference is given as a step function of $1 \mathrm{~m}$. Then, from 10s, the disturbance is also inputted as a step function of $50 \mathrm{~N}$. The cutoff-frequency of the local-side DOB is set to $100 \mathrm{~Hz}$ (about $600 \mathrm{rad} / \mathrm{s}$ ).

As same with the bode-plot in Fig. 3a, the final value of the estimation error given by the output is zero. It is validated from Fig. 6a, 7a, 8a, and 9a. On the other hand, about the disturbance response shown in Fig. $6 \mathrm{~b}$ and $7 \mathrm{~b}$, although the case 3 has the large steady-state estimation-error, the case 4 can suppress the error by introducing the frequencyweight includes an integral. The reason is that the steady-state estimation error per $1 \mathrm{~N}$ of case 3 and 4 shown in Fig. 3b is about $-0.3 \mathrm{~m} / \mathrm{N}$ and $-1.3 \times 10^{-14} \mathrm{~m} / \mathrm{N}$, respectively. On the other hand, the disturbance is $50 \mathrm{~N}$. Then, the error in case 3 and 4 becomes about $-15 \mathrm{~m}$ and $-2 \times 10^{-14} \mathrm{~m}$ as shown in Fig. $6 \mathrm{~b}$ and $7 \mathrm{~b}$. Meanwhile, in Fig. $8 \mathrm{~b}$ and 9b, implementing the local-side DOB, the influence of the disturbance is enough suppressed and the steady-state error is decreased much. The peak estimation-error since $10 \mathrm{~s}$ is depending on the cutofffrequency of the local-side DOB.

From these results and a comparison of the estimation error shown in Fig. 10, it is validated that the case 4 under local DOB has most robustness to the disturbance. Due to these comparison, the robustness of the proposed NCS is better than the conventional design.

\section{Conclustion}

This paper presents the robustness improvement of the LKF based $\mathrm{CDOB}$, by introducing the frequency-weight for the disturbance-input. To suppress the estimation error of the CDOB given by the disturbance, high gained LPF and integral factor are introduced as the weight. When the LPF is only applied, depending on the amplitude of the disturbance, it is difficult to neglect the steady-state error. On the other hand, introducing the LPF with integral, the error suppression is much improved by the infinite gain in steady-state. Additionally, implementing the local-side DOB for the disturbanceinput suppression, the error is also much more suppressed. From the numerical simulations, the account of the authors' proposal is validated.

In future works, experiments are conducted to validate the results. Additionally, considering the performance of local-side $\mathrm{DOB}$, the conservativeness of the $\mathrm{CDOB}$ would be decreased.

\section{ACKNOWLEDGMENT}

We thank to Prof. Uchimura in Shibaura Institute of Technology for many advices to construct the LMI of the LKF based CDOB.

\section{REFERENCES}

[1] R. Drauth and A. Horch, "Industrie 4.0: Hit or hype?," IEEE Industrial Electronics Magazine, vol. 8, no. 2, pp. 56-58, 2014.

[2] T. Shimono, S. Katsura, and K. Ohnishi, "Abstraction and Reproduction of Force Sensation From Real Environment by Bilateral Control," in IEEE Transactions on Industrial Electronics, vol. 54, no. 2, pp. 907918, 2007.

[3] M. Franken, et al, "Bilateral Telemanipulation With Time Delays: A Two-Layer Approach Combining Passivity and Transparency," in IEEE Transactions on Robotics, vol. 27, no. 4, pp. 741-756, 2011.

[4] D. Gunji, T. Imura, and H. Fujimoto, "Fundamental research of power conversion circuit control for wireless In-Wheel Motor using magnetic resonance coupling," IECON 2014 - 40th Annual Conference of the IEEE Industrial Electronics Society, pp. 3004-3009, 2014.

[5] Y. Shi, J. Huang, and B. Yu, "Robust Tracking Control of Networked Control Systems: Application to a Networked DC Motor," in IEEE Transactions on Industrial Electronics, vol. 60, no. 12, pp. 5864-5874, 2013.

[6] J. Qiu, H. Gao, and S. X. Ding, "Recent Advances on Fuzzy-ModelBased Nonlinear Networked Control Systems: A Survey," in IEEE Transactions on Industrial Electronics, vol. 63, no. 2, pp. 1207-1217, 2016.

[7] C. L. Lai and P. L. Hsu, "Design the Remote Control System With the Time-Delay Estimator and the Adaptive Smith Predictor," in IEEE Transactions on Industrial Informatics, vol. 6, no. 1, pp. 73-80, 2010.

[8] K. Natori, et al, "Time-Delay Compensation by Communication Disturbance Observer for Bilateral Teleoperation Under Time-Varying Delay," in IEEE Transactions on Industrial Electronics, vol. 57, no. 3, pp. 10501062, 2010.

[9] E. Fridman, "New LyapunovKrasovskii functionals for stability of linear retarded and neutral type systems," Systems and Control Letters, vol. 43, issue 4, pp. 309-319, 2001. 


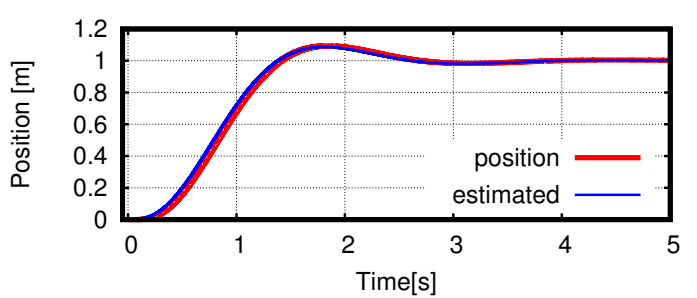

(a) until $5 \mathrm{~s}$, the reference is $1 \mathrm{~m}$

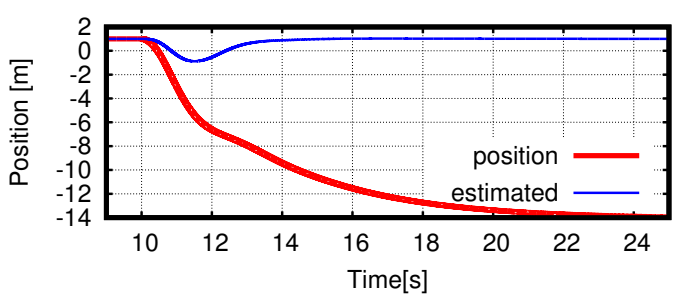

(b) since $10 \mathrm{~s}$, the disturbance $(50 \mathrm{~N})$ is inputted

Fig. 6: The LKF based CDOB using the frequency-weight of case 3, without local-side DOB.

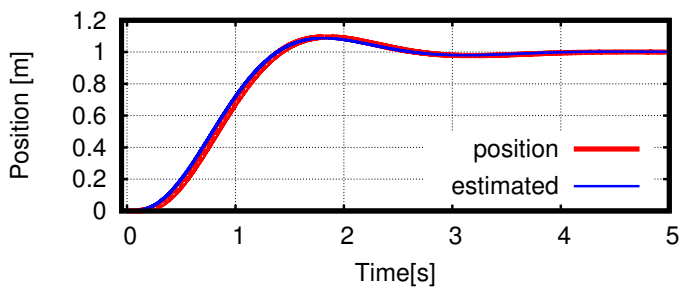

(a) until $5 \mathrm{~s}$, the reference is $1 \mathrm{~m}$

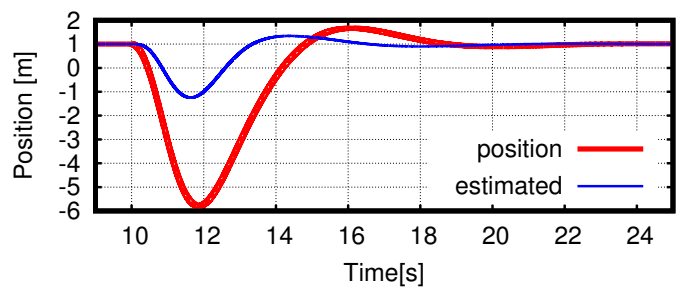

(b) since $10 \mathrm{~s}$, the disturbance $(50 \mathrm{~N})$ is inputted

Fig. 7: The LKF based CDOB using the frequency-weight of case 4, without local-side DOB.

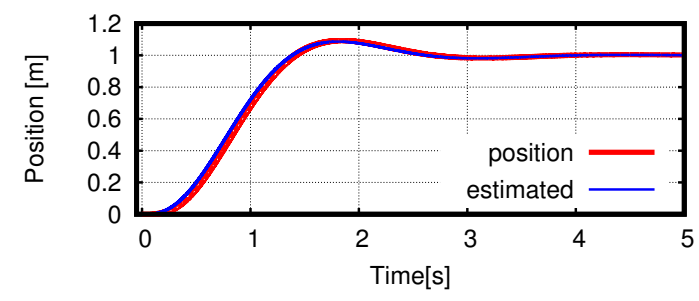

(a) until $5 \mathrm{~s}$, the reference is $1 \mathrm{~m}$

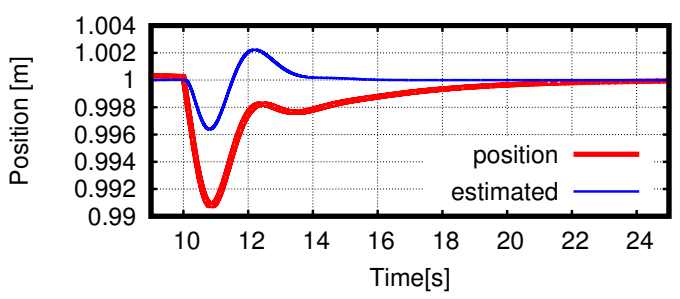

(b) since $10 \mathrm{~s}$, the disturbance $(50 \mathrm{~N})$ is inputted

Fig. 8: The LKF based CDOB using the frequency-weight of case 3, with local-side DOB.

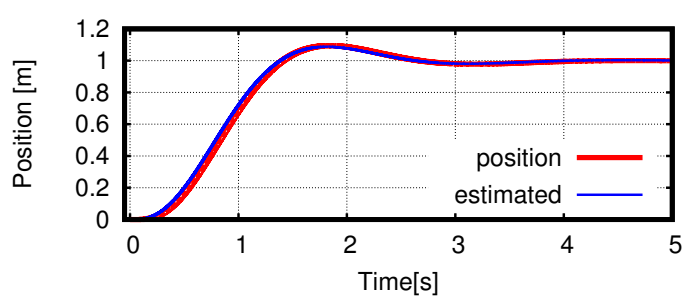

(a) until $5 \mathrm{~s}$, the reference is $1 \mathrm{~m}$

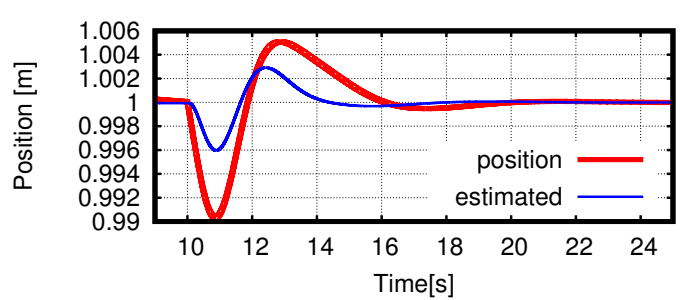

(b) since $10 \mathrm{~s}$, the disturbance $(50 \mathrm{~N})$ is inputted

Fig. 9: The LKF based CDOB using the frequency-weight of case 4, with local-side DOB.

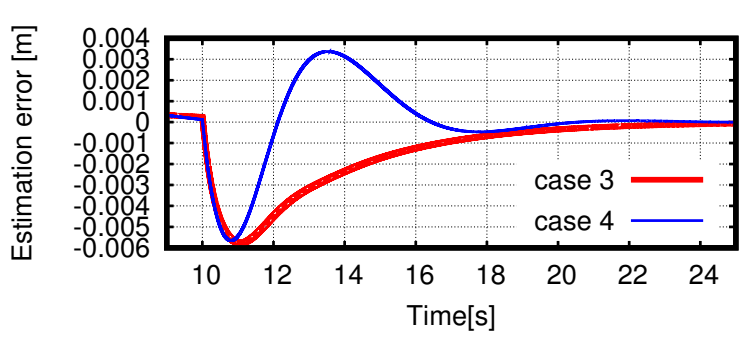

Fig. 10: The estimation error in Fig. $8 \mathrm{~b}$ and $9 \mathrm{~b}$.

[10] E. Fridman, "Stability of systems with uncertain delays: a new" complete" Lyapunov-Krasovskii functional," in IEEE Transactions on Automatic Control, vol. 51, issue 5, pp. 885-890, 2006.

[11] X. Jiang and Q. Han, "On $\mathrm{H} \infty$ control for linear systems with interval time-varying delay," Automatica, vol. 41, pp. 2099-2106, 2005.

[12] H. B. Zeng, et al, "Free-Matrix-Based Integral Inequality for Stability Analysis of Systems With Time-Varying Delay," in IEEE Transactions on Automatic Control, vol. 60, no. 10, pp. 2768-2772, 2015.

[13] N. Uematsu, T. Suhara, and Y. Uchimura, "Model-Error Feedback for Systems with Time-Varying Delay Based on Mixed H2/H $\infty$ Control," IEEJ Journal of Industry Applications, vol. 5, no. 3, pp. 245-252, 2016.

[14] Y. Uchimura, "Stability and Stabilization of Systems with Time Delay," IEEJ Journal of Industry Applications, vol. 5, no. 4, pp. 321-328, 2016.

[15] M. Koyama and Y. Asano, "Improvement in Precision of Positioning Control System via $2.4 \mathrm{GHz}$ Band WirelessCommunication," IEEJ Transactions on Industry Applications, vol.137, no.7, pp.553-560, 2017.(in Japanese) 